\title{
Use of Robotic Telepathology for Frozen-Section Diagnosis: A Retrospective Trial of a Telepathology System for Intraoperative Consultation
}

\author{
Keith J. Kaplan, M.D., Jeanette R. Burgess, M.D., Glenn D. Sandberg, M.D., Cris P. Myers, M.D.,
} Thomas R. Bigott, B.S., Renata B. Greenspan, M.D.

Department of Pathology (KJK, JRB, RBG) and Telemedicine Directorate (TRB), Walter Reed Army Medical Center, Washington, DC; Department of Neuropathology, Armed Forces Institute of Pathology (GDS), Washington, DC; and Department of Pathology, Heidelberg Army Hospital (CPM), Heidelberg, Germany

Telepathology is the practice of digitizing histological or macroscopic images for transmission along telecommunication pathways for diagnosis, consultation, or continuing medical education. Previous studies have addressed static versus dynamic imaging in several specimen types with a wide variety of systems and communication pathways. The goal of this paper was to assess the validity of a Web-based telepathology system for frozen section consultation within the Army Medical Department. The system provides real-time, dynamic remote control of a robotic microscope over standard Internet connections. Oftentimes, a solo pathologist is called on to provide diagnostic services without the support of immediate second or expert consultation during an intraoperative consultation. The use of telepathology is attractive because it provides an opportunity for pathologists to obtain immediate consultation. For purposes of the study, 120 consecutive frozen section cases were diagnosed at a distance using the system. Intraobserver agreement between the telepathology diagnosis and glass slide diagnosis was observed. Diagnostic agreement was $100 \%$ for a wide variety of specimens. This study suggests that such a system will help support pathologists located at distant sites.

\footnotetext{
Copyright () 2002 by The United States and Canadian Academy of Pathology, Inc

VOL. 15, NO. 11, P. 1197, 2002 Printed in the U.S.A.

Date of acceptance: July 20, 2002.

This project was funded by Telemedicine \& Advanced Technology Re search Center (TATRC)/United States Army Medical Research and Materiel Command (USAMRMC) HSRRB Log No. A-10244.

The selection, installation, and use of the Trestle Corporation MedMicroscopy system does not imply an endorsement thereof. The opinions and assertions contained herein are the private views of the authors and are not to be construed as official or as reflecting the official views of the United States Department of the Army or Department of Defense. Address reprint requests to: Keith J. Kaplan, M.D., Department of Pathology, Walter Reed Army Medical Center, 6900 Georgia Avenue NW, Washington, DC 20307-5001; e-mail: keith.kaplan@na.amedd.army.mil; fax 202-782-3217
}

DOI: $10.1097 / 01 . M P .0000033928 .11585 .42$
KEY WORDS: AFIP, Diagnosis, Frozen section, Telepathology.

Mod Pathol 2002;15(11):1197-1204

Telepathology is the practice of digitizing histological or macroscopic images for transmission along telecommunication pathways for diagnosis, consultation, or continuing medical education. In dynamic (real-time) telepathology, the consultant examines a slide remotely with a robotic microscope that allows him or her to select different fields and magnification powers. Static telepathology relies upon images sent by the referring pathologist. Weinstein and colleagues (1) reported the progress of telepathology previously. Singson et al. (2) showed the first use of telepathology via the Internet in 1989. In the same year, investigators in Norway were the first to provide intraoperative frozen section (FS) services to several rural hospitals via telepathology (3). Since that time, there have been several articles published in the literature. The number of telepathology abstracts indexed in MEDLINE has grown from $<5$ in 1986 to nearly 100 in 2000. Several of these studies deal with a single organ system or in practices with a limited scope of pathologic specimens (4-16).

A few telepathology services are active in the United States, including those of the Armed Forces Institute of Pathology (AFIP), the University of Arizona, and the Milwaukee Veterans Administration Medical Center (12). For a variety of reasons, telepathology has not gained wide acceptance among practicing pathologists. The Military Health Care System (MHCS) is the ideal setting for telepathology services, with several solo pathologists throughout the world, isolated from regional medical centers.

One of the most promising applications of telepathology is intraoperative consultation (FS diagnosis) to be allowed between small hospitals with 
pathology support located elsewhere, allowing operations requiring an intraoperative histopathological diagnosis pathology support without a pathologist on site. Telepathology can also be used to support an isolated pathologist for second or expert consultation or even to completely transfer the diagnostic work to another facility, as previously described by Dunn et al. (17).

Robotic telepathology provides remote consultation via a remote microscope that can be controlled by the consulting pathologist. Because the field selection is accomplished by the consultant, the information that he or she obtains, except for digital imaging capabilities, is the same as he or she would obtain at the microscope in person.

Robotic telepathology for FS analysis and diagnosis should enable remote sites without direct pathologic support to obtain needed expert consultation intraoperatively, thereby preventing medical errors, reducing costs, and increasing quality and access for military personnel and their families.

In the worldwide MHCS, continued physician downsizing is mandating a shift from medical support specialties. It is becoming increasingly necessary to employ technology to utilize these laboratory medicine specialists more effectively. No longer can the economies of scale support a single pathologist at small Army community hospitals. The loss of a pathologist means a sharp curtailment in the extent of surgical services that can be rendered at these community-sized hospitals. The request for consultation to an outside source is a standard procedure in surgical pathology practice. In particular, members of small pathology departments suffer from lack of "next-door" specialists and consequently have a greater need to submit histological slides to a remote pathologist. This requires packing and postage expenses, particularly if material has to be sent transcontinentally via private couriers. Additionally, increasing documentation between countries is necessary to ensure the lack of biological risk associated with the submitted material.

The use of remote pathology may allow full surgical pathology support to remote centers by pathologists located at distant sites. A validated remote pathology system, which meets community standards of diagnostic accuracy, will help support the current number of pathologists while maintaining microscopic diagnostic support to communitysized hospitals and regional medical centers. Telepathology would enable full-time coverage and would reduce the travel time of the pathologist, which is expensive, nonproductive professional time.

This study evaluated the feasibility and effectiveness of transmitting FS slide images from a remote site to Walter Reed Army Medical Center (WRAMC)
Department of Pathology, Heidelberg Army Hospital (HAH) Department of Pathology, or the AFIP. This is the first time that any pathology project of this nature has been performed in the Army Medical Department (AMEDD).

\section{METHODS AND MATERIALS}

\section{Study Design}

We retrospectively analyzed 120 cases. The telepathologist was given the usual clinical information with regard to organ site, sex, and age of the patient and any pertinent clinical or radiographic information that was available on the surgical pathology tissue examination request form at the time of the intraoperative consultation.

Each case was evaluated by telepathology without prior knowledge of the FS or final diagnosis and could be given a diagnosis or deferred if image or technical quality was suboptimal. All slides that were used in the telepathology evaluation were later reviewed by conventional light microscopy by the same pathologist to assess intraobserver agreement between the telepathology and FS diagnosis. Later, all diagnoses were compared with the original FS and final diagnosis to assess interobserver agreement. Diagnostic errors were classified as interpretation errors if differences between telepathology and FS diagnosis were obtained and as sampling errors if discrepancies between the FS slides and slides prepared from formalin-fixed, paraffin-embedded tissues were seen. Agreements between the telepathology and glass slide diagnosis were reviewed and compared with the final diagnosis in conjunction with routine monthly intradepartmental quality assurance programs (new FS diagnosis versus final diagnosis).

Diagnostic errors were further classified as either clinically significant or insignificant. Discrepancy cases were evaluated by two independent pathologists (those who did not participate in the telepathology, FS, or final diagnosis assessment).

One of three board-certified Anatomic/Clinical pathologists in the Departments of Pathology at WRAMC, HAH, and the AFIP was responsible for all telepathology case transmissions.

Remote diagnosis was carried out between referring institutions to WRAMC, HAH, and the AFIP. The Trestle Corporation (Newport Beach, CA) MedMicroscopy system was initially installed at three referring institutions throughout the AMEDD. Initial installation sites include Walter Reed Army Medical Center (Washington, DC), Ireland Army Community Hospital (Fort Knox, KY), Womack Army Medical Center (Fort Bragg, NC), and Landstuhl Regional Medical Center (Landstuhl, Germany). Cases were transmitted from the daily work- 
load throughout the AMEDD. The use of consecutive cases eliminated bias related to case complexity. All slides used in the FS by the host site (e.g., Fort Knox or WRAMC) were sent to the remote site (e.g., WRAMC or AFIP) after final review and diagnosis of the permanent (paraffin) sections at the host site and transmitted to the surgeon in the usual fashion. The remote consultation was not carried out as part of the intraoperative consultation or permanent section final diagnosis. The consulting pathologists at WRAMC, HAH, and AFIP were blinded as to the diagnosis at the host site. Thus, after evaluation at the host site, retrospective review of the telepathology images and FS glass slides was done at the remote site for purposes of validation of this technology in the AMEDD. FS diagnoses were given by 1 of 11 pathologists. None of the pathologists rendering a FS diagnosis by conventional microscopy participated in the telepathology evaluation. Therefore, FS slides were examined separately by a pathologist using telepathology.

The pathologist at the remote site evaluated the telepathology slides and rendered a telepathology diagnosis. Upon receipt of the FS slides, the consulting telepathologist reviewed and rendered a diagnosis. This diagnosis was compared with the telepathology diagnosis to assess intraobserver agreement. Correlation between the telepathology diagnosis, FS diagnosis, and final diagnosis was done only for purposes of quality assurance between the FS diagnosis and permanent section diagnosis and to assess interobserver agreement.

All clinical information regarding the sample (patient age, sex, medical history, surgical procedure, etc.) as given to the host site was made available to the remote site pathologist.

A text diagnosis, time to make the diagnosis, as well as the number of slides reviewed, were recorded. The remote site rendered an evaluation limited to the following parameters:

a. Definitive diagnosis

b. Deferred diagnosis pending glass slides based on:

1. Specimen sampling/adequate numbers of slides

2. Technical quality of the telepathology system

In addition to validation of diagnostic agreement of the telepathology system, each pathologist subjectively assessed the image quality and technical quality of the system. This was to be graded on a four-tier scale, with a score of 1 being the lowest score (poor) and a score of 4 being the highest score (excellent) according to the following criteria:

\section{Image Quality}

1. Images extremely distorted, precluding any pathologic evaluation via telepathology. Images are of such poor quality that no diagnosis can be ascertained from such images via telepathology.

2. Images contain excess or loss of pixels or fields of view that are difficult to evaluate. Loss of highquality resolution precludes an accurate diagnosis via telepathology.

3. Images are of high-quality resolution with nearly flawless transmission. Images are of high enough quality as to render a diagnosis via telepathology.

4. Flawless transmission of high-quality resolution images. Allows real-time, visually lossless performance to render a diagnosis via telepathology.

\section{Technical Quality}

1. Connection lost or unstable precluding any pathologic evaluation via telepathology. Transmission of such poor quality that no diagnosis can be ascertained via telepathology.

2. Connection slow or unstable image processing, instrument manipulation, or data storage, precluding any pathologic evaluation via telepathology. Transmission of all fields difficult to evaluate. Loss of high-quality transmission precludes an accurate diagnosis via telepathology.

3 . Nearly flawless transmission and image processing. Transmission is of high enough quality as to render a diagnosis via telepathology.

4. Flawless transmission, image processing, instrument manipulation, and data storage into a seamless core. Allows real-time, visually lossless performance to render a diagnosis via telepathology.

The primary objective of this study was to compare the diagnostic accuracy of telepathology for FS diagnoses as compared with conventional light microscopy for FS diagnoses. The primary outcome variable in this study was the coded diagnosis. For each diagnostic session, the telepathology diagnosis was recorded, along with the time to diagnosis (TTD). This diagnosis was later compared with the FS diagnosis on evaluation of the glass slides used for the telepathology session.

On-site training was provided by the vendor in conjunction with the project manager for this study from the WRAMC Department of Telemedicine and the pathologists at all the host and remote sites.

\section{Statistical Analysis}

For each diagnostic session, the telepathology diagnosis and FS diagnosis were recorded along with the TTD for the telepathology session. When software problems occurred, a description of the problem was recorded. Such reports were forwarded to the vendor to improve the system.

Diagnostic agreement has been described as percentage of corresponding FS and telepathology diagnosis (similar coded text diagnoses with identical 
clinical significance); the precision of estimate for diagnostic accuracy has been given by means of approximate $95 \%$ confidence intervals (CI), as suggested by Harper and Reeves (18). TTD was recorded for each case to the nearest quarter minute and summarized with descriptive statistics.

Other qualitative data included the acceptability of the system in terms of image quality and overall technical performance. Each diagnostic session required the telepathologist to evaluate the image and technical quality for each slide reviewed. These were evaluated in a semiquantitative fashion using the previously described scale.

\section{Equipment and Specifications}

This study used the MedMicroscopy telepathology system and software manufactured and developed by the Trestle Corporation (Newport Beach, CA) (Fig. 1). The system consists of a microscope site, where the MedMicroscopy software is installed on a standard PC connected to an automated microscope, and a viewer site that consists of a standard PC running MedMicroscopy viewer software. The systems communicate using Transmission Control Protocol and Internet Protocol (TCP/IP), allowing users to communicate over standard Internet/Intranet lines.

The microscope site consists of the following components: 1) software, 2) microscope, 3) automation kit, 4) video components, and 5) PC workstation. These in turn include the following:

1. Software: MedMicroscopy v2.0 (Trestle Corporation)

2. Microscope: Olympus BX50 with 4X, 10X, 20X and 40X objectives and 10X eyepieces (Olympus America, Melville, NY).

3. Automation kit: motorized nosepiece (Olympus America); motorized stage (Prior Scientific Inc., Rockland, MA); controller box (Trestle Corporation).

4. Video components: Panasonic GPKS1000 camera (Matsushita Electric Corporation of America, Secaucus, NJ); PE photo eyepiece (Olympus America); PCI Bus RGB color frame grabber (Imaging Technologies Corporation, San Diego, CA).

5. PC Workstation: Dell Precision 220 computer with a Dell 21-inch monitor (Dell Computer Corporation, Austin, TX) running Windows NT Server (Microsoft Corporation, Redmond, WA).

The viewer site consists of any Windowscompatible PC $(98 / 2000 / \mathrm{ME} / \mathrm{XP} / \mathrm{NT})$ with a Dell 17-inch monitor running MedMicroscopy viewer software with an IP connection to the microscope site.

The MedMicroscopy system is not a video transfer system. Conventional video conferencing systems used in telepathology have a limited $355 \times 288$ image resolution. MedMicroscopy uses a unique real-time, image update mechanism that sends new images only when a new movement (or change in state) of the microscope is completed (on selection of field or magnification). This delivers very highquality images that are three times the resolution of video teleconferencing $(352 \times 288=101,376$ pixels versus $640 \times 480=307,200$ pixels). Images are compressed for transmission using industry standard JPEG compression.

This technique has the advantage of using significantly less bandwidth than in the case of video because new images at $40-60 \mathrm{~K}$ in JPEG format at $640 \times 480$ are sent only when needed. Typically, this technology requires 3 -fold less bandwidth than video technology. Additionally, this technology can easily accommodate higher resolution cameras as they become available, for example, a $1280 \times 1024$ resolution adapts to this system. Because of MedMicroscopy's static-based image update mechanism (as described above), image quality is independent of bandwidth. Recommended system bandwidth is 200-400 Kbps; users at lower bandwidths experience the same image quality, only with longer latency between images.

The software captures high-fidelity color images. In its standard configuration, MedMicroscopy uses individually sampled red, green, and blue outputs from a high-resolution, high-density, 900,000 pixel, charge-coupled device color camera that produces 560 lines of resolution to produce a high-resolution $(640 \times 480), 24$-bit true-color image. Use of red, green, and blue sampling produces very high color fidelity relative to such sampling methods as composite and S-video, which video conferencing technology relies on.

The viewer sites used 17 -inch monitors with a native resolution of $1024 \times 768$ and 24-bit true color. Color calibration between the microscope site and viewer site was not routinely performed during each telepathology session.

\section{RESULTS}

One hundred twenty cases were accrued into the study that represented several organ systems reflective of the surgical pathology workload within the AMEDD (Table 1).

Ninety of 120 (75\%) of the cases were for primary diagnosis (unknown disease process). The remaining 25\% were FSs for staging procedures or extent of resection (Table 2). Diagnostic concordance was determined by having similar coded text diagnoses with identical clinical significance. The diagnostic agreement between the telepathology and FS diagnoses was $100 \%$ (CI: $97.5-100 \%$ ). Of note, in two cases, the pathologists' telepathology and FS diag- 
TABLE 1. Distribution of Organ Systems Involved in FS Consultations $(n=120)$

\begin{tabular}{lc}
\hline Organ/Site & No. of Cases \\
\hline Adrenal & 1 \\
Bladder & 3 \\
Brain & 14 \\
Cervix & 1 \\
Colon & 5 \\
Kidney & 1 \\
Larynx/pharynx & 17 \\
Liver & 2 \\
Lung & 15 \\
Lymph node & 13 \\
Omentum & 2 \\
Ovary & 19 \\
Pancreas & 1 \\
Parathyroid & 5 \\
Parotid & 1 \\
Small bowel & 1 \\
Soft tissue & 4 \\
Testicle & 3 \\
Thymus & 3 \\
Thyroid & 5 \\
Uterus & 4 \\
\hline
\end{tabular}

TABLE 2. Distribution of Cases for FS Consultation ( $n=$ 120)

\begin{tabular}{cc}
\hline Case Composition & No. of Cases \\
\hline Primary diagnosis & 75 \\
Nonneoplastic diseases & 8 \\
Benign neoplasms & 45 \\
Malignant neoplasms & 22 \\
Staging procedures & 12 \\
Extent of resection (margins) & 18 \\
\hline
\end{tabular}

noses were concordant but were not in agreement with the final diagnosis. In each case, the error was attributable to sampling or technical error that would not have been clinically significant. The same pathologist generated these errors. In both of the cases, the telepathology diagnosis was concordant with the FS diagnosis, as would be expected in the case of sampling error. Both of these cases involved ovarian masses for primary diagnosis. In one case, a hemorrhagic cyst was better classified as endometriosis after additional sampling, and clinical information not given at the time of intraoperative consultation was obtained. In the other case, a benign serous cyst was better classified as a luteinized cyst. These cases were further evaluated by two independent pathologists (those who did not participate in the telepathology, FS, or final diagnosis assessment) who agreed with the FS and final diagnosis.

The overall median TTD was 2 minutes, $50 \mathrm{sec}-$ onds (50 seconds to 9 minutes). The longest time recorded was 9 hours, for an open-lung biopsy that covered approximately $40 \%$ of the slide surface and required review of multiple high-powered fields because of the focal nature of the lesion in a case with scattered granulomas that were not clinically suspected (Fig. 2). The shortest time recorded was 50 seconds, for a ureteral margin on a cystectomy specimen that consisted of a narrowed lumen measuring $0.2 \mathrm{~cm}$ in greatest diameter.

Other qualitative data included the suitability of the image quality and overall technical performance. The image quality was rated as " 4 " or "excellent" in $98 \%$ of the cases with excellent cytologic and nuclear detail (Fig. 3). The remaining two cases were rated "3" ("good") and "2" ("fair"). The overall technical quality of the system was rated as " 4 " ("excellent") in $97 \%$ of the cases. On two occasions, software problems with the remote computer resulted in reestablishing the connection with the host site, resulting in a delay of 45 seconds for each case before initiating the telepathology consultation. On one occasion the telepathology session was terminated before diagnosis because of an unstable Internet connection between WRAMC and Heidelberg. Restart and reconnection with the server site was successful, with nearly flawless transmission of images between the two sites. No diagnoses were deferred pending glass slides based on specimen sampling or adequate numbers of slides or on the technical quality of the telepathology system. Several pathologists within the Department of Pathology at WRAMC informally evaluated the system with intradepartmental cases with "excellent" overall evaluations after a brief orientation to the system using the hospital local area network. Additionally, five cases were successfully transmitted to other remote locations (pathologists' residences) using Pentium III PCs over standard Internet connections using commercial Internet service providers at speeds ranging from 33.6 to $52 \mathrm{kbps}$.

The main complaint was the period of time required to screen multiple pieces or a single large piece of tissue at high power. Additionally, familiarity and diagnostic confidence using the system varied between the three pathologists participating in the study. On average, diagnostic confidence with the system was high after six telepathology diagnosis sessions.

\section{DISCUSSION}

FS diagnosis has been used as an important factor in intraoperative decision making since the end of the 19th century (19). Welsh (quoted by Remsen et al. [20]) first used FS as a method when he froze a breast lesion removed by Halsted. FS is technically limited and more difficult to interpret than examination of formalin-fixed, paraffin-embedded sections. Nevertheless, FS is regarded as an accurate means of diagnosis during surgery and often has a significant influence on the surgical operation being performed. The major indications for performing a FS are to obtain a diagnosis of a patho- 


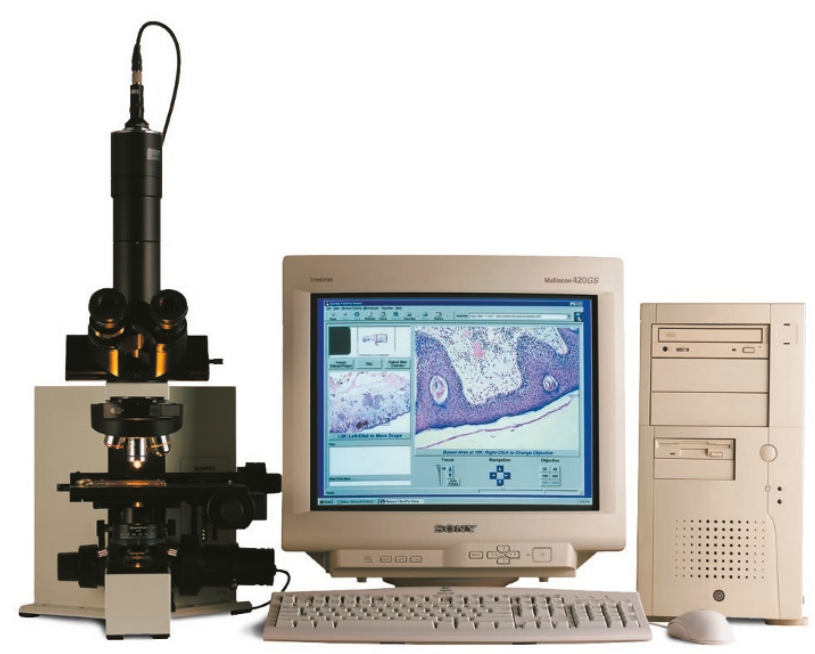

FIGURE 1. MedMicroscopy server site.

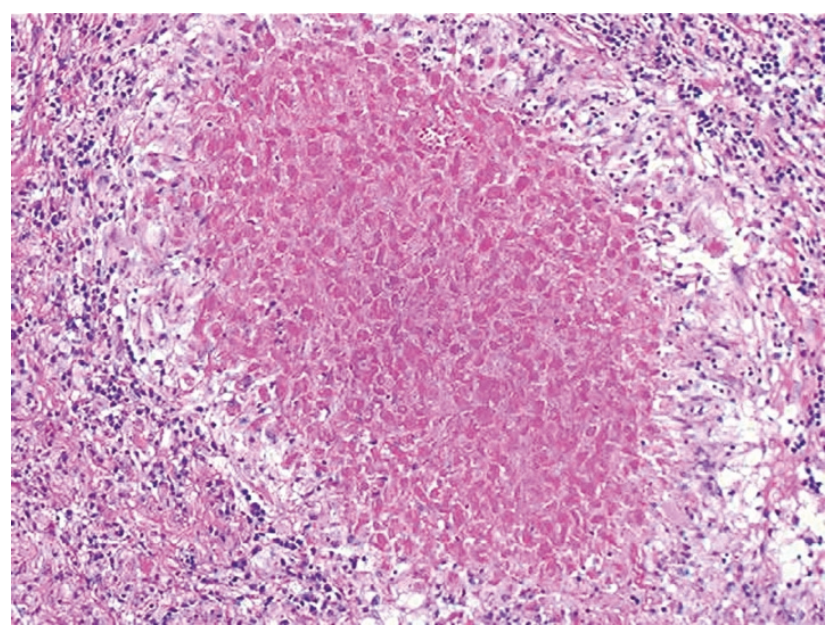

FIGURE 2. Necrotizing granuloma within a lung biopsy specimen. (Lung FS, H\&E; 20×;TIF)

logic process, determine presence or absence of tumor metastases in lymph nodes, or determine adequacy of resection margins of a malignant lesion. The accuracy of FS diagnosis reported in the literature varies between $94 \%$ and $97.4 \%$. Some of the results reported are combinations of different types of tissue submitted for FS (21-23), whereas others reflect specific tissue examined (19, 24-28).

The diagnostic agreement in our study is comparable to rates of agreement $(92-100 \%$ accuracy) in several recently published telepathology studies of FS, permanent section, and cytological slides $(2,20$, 29-31). Dunn et al. reported telepathology concordance for clinically important diagnoses compared with light microscopy by the same pathologist was achieved in 2138 of 2144 cases (99.7\%) (17). In a recent meta-analysis assessing the reliability of telepathology for FS service, a total of $>1290$ cases showed a slightly lower overall diagnostic accuracy of about 0.91 than the conventional FS with an average accuracy of about 0.99 found in an analysis

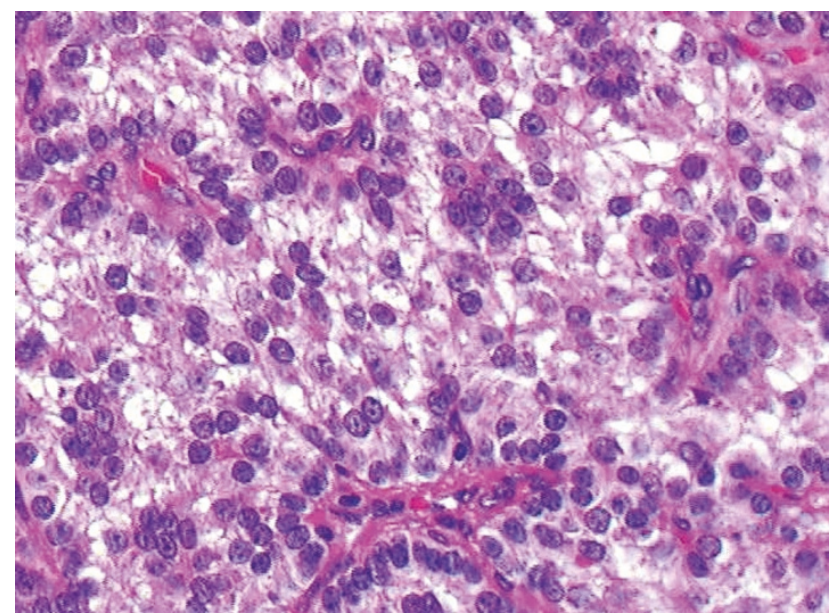

FIGURE 3. Carcinoma with neuroendocrine features. Note rosettes and peripheral arrangement of nuclei with eosinophilic granular cytoplasm and finely granular chromatin (lung FS, H\&E; 40×;TIF).

of several studies of different organs (32). The difference is at least predominantly caused by a higher rate of deferral and false-negative FS diagnoses in the telepathology method, whereas the specificity of both methods, each $>0.99$, was not clinically different.

Prospective studies on the diagnostic validity of telepathological FS diagnosis have previously been performed by several European (32-34), Scandinavian $(3)$, and Japanese $(4,35)$ investigators with a variety of telecommunication and telepathology systems. In the United States, Winokur et al. (12) prospectively reviewed 99 intraoperative consultations from 29 tissue/organ sites. Statistical analysis indicated no significant difference between telepathology and conventional microscopy. In several previous studies, the use of telepathology for diagnostic purposes has been validated in the surgical pathology laboratory (5-7). Two international static telepathology consultative services have shown diagnostic accuracy rates of $88.2 \%$ and $100 \%$, with an emphasis on the importance of the telepathologist to establish "diagnose versus defer" decisions on consultation cases for static telepathology (2).

Diagnostic accuracy rates for pathologists are higher with dynamic (real-time, robotic) telepathology than for static telepathology. In dynamic telepathology, the consultant examines a slide remotely with a robotic microscope that allows him or her to select different fields and magnification powers, thereby replicating routine use of a microscope from a remote site. In contrast, static telepathology relies upon images sent by the referring pathologist who transmits selected images for diagnosis by the remote pathologist; this process is more prone to sampling errors by the referring pathologist.

Misdiagnoses in the telepathological examination of FSs can be attributed to four kinds of error: 
selection of an inappropriate tissue sample for frozen sectioning (sampling error); selection and transmission of inappropriate microscopic fields, especially in the case of static telepathology; misinterpretation despite adequate images of sufficient quality; and misinterpretation due to images of insufficient quality (32). Although misinterpretation is not related to a given telepathology diagnostic session, it may be caused by or imposed by telepathology, causing decreased diagnostic accuracy. Previous studies concerning the use of telepathology for FS diagnosis do not address the indication for FS (i.e., primary diagnosis, extent of resection [margins], or staging procedures). Similarly, it is unclear whether selected or unselected cases were examined for study.

For telepathology to be successful, the laboratory must ensure that the remote pathologist has adequate access to appropriate diagnostic material. In a practice setting in which primary telepathology diagnoses are rendered and a pathologist is not present at a referring site, a methodology must be employed that ensures that video sampling of gross tissues and glass slides is inclusive. With the current system used in this study, the vendor manufactures such a gross station to allow the remote site visual access to the gross specimen from which slides were prepared that is equipped with a video camera for streaming live video, whereby a pathologist can select the tissue for FS diagnosis. This technology was not assessed or validated, as this was a retrospective study. A gross description of the lesion was provided to the telepathologist at the time the slides were reviewed. No macroscopic images were transmitted.

The gross examination of the tissue sample by a nonpathologist in a primary telepathological diagnostic session assumedly increases the risk of selection of inappropriate tissue for the microscopic examination. A study to verify and estimate the significance of this source of error is difficult to design. The cited retrospective studies do not allow conclusions on the significance of this source of error because they do not show a trend to higher levels of diagnostic accuracy compared with the prospective studies (32). Some telepathology systems rely on the transmission of macroscopic images and subsequent visual and acoustic guidance of tissue sampling by the telepathologist with the aid of technician, pathologist assistant, or surgeon $(3,12)$. In the fields of microinvasive surgery, computer-assisted surgery, and telesurgery, sensors that allow for tactile sensation (i.e., telefeeling, telepalpation) are being developed that may be suitable for the tactile sensation needed for a remote telepathologist for FS diagnosis (32). Of note, in traditional second-opinion pathology, oftentimes a gross photograph or specimen may not be available to the consulting pathologist, who may need to rely upon the gross description supplied by the referring pathologist.

In addition to assuring that the remote pathologist has access to adequate diagnostic material, laboratories involved in primary-diagnosis or secondopinion telepathology must identify the case appropriately; ensure that all appropriate clinical information is conveyed to the remote pathologist; and have procedures for data handling, archiving, and security. Because the effectiveness and accuracy of telepathology depend critically on the skill and judgment of the pathologist, both referring and consulting pathologists practicing telepathology should be trained in telepathology. Training should occur on the individual systems that the pathologist uses and on general telepathology and imaging principles and limitations. Specific laboratory guidelines should be driven by best clinical practices in conventional laboratory procedures. The complexity associated with these issues is beyond the scope of this study.

\section{CONCLUSIONS}

This study validates the use of telepathology for FS diagnosis, which meets community standards of diagnostic accuracy and can help support the reduced numbers of pathologists, while maintaining pathology support to surgical services at community-sized hospitals or hospitals lacking fulltime pathology coverage.

Future studies will evaluate the effectiveness and feasibility for this technology to support real-time telepathology consultation between smaller hospitals, regional medical centers and the AFIP. In the future, clinically approved protocols will be developed for the provision of pathologic care using telepathology for routine, emergent, and FS diagnosis. This will support primary and secondopinion pathology diagnosis throughout the worldwide MHCS.

\section{REFERENCES}

1. Weinstein RS, Bhattacharyya AK, Graham AR, et al. Telepathology: a ten-year progress report. Hum Pathol 1997;28:1-7.

2. Singson RP, Natarajan S, Greenson RK, et al. Virtual microscopy and the Internet as telepathology consultation tools. A study of gastrointestinal biopsy specimens. Am J Clin Pathol 1999;111:792-5.

3. Nordrum I, Eide TJ. Remote frozen section service in Norway. Arch Anat Cytol Pathol 1995;43:253-6.

4. Shimosato Y, Yagi Y, Yamagishi K, et al. Experience and present status of telepathology in the National Cancer Center Hospital, Tokyo. Zentralb Pathol 1992;8:413-7.

5. Weinberg DS, Allaert FA, Dussere P, et al. Telepathology diagnosis by means of digital still images: an international validation study. Hum Pathol 1996;27:111-8. 
6. Weinstein MH, Epstein JI. Telepathology diagnosis of prostate needle biopsies. Hum Pathol 1997;28:22-9.

7. Weinstein LJ, Epstein JI, Edlow D, et al. Static image analysis of skin specimens: the application of telepathology to frozen section evaluation. Hum Pathol 1997;28:30-5.

8. Dunn B, Almagro U, Choi H, et al. Dynamic-robotic telepathology: Department of Veterans Affairs feasibility study. Hum Pathol 1997;28:8-12.

9. DeYoung BR, Niemann TH, Hitchcock CL. Using telepathology to assess frozen sections of breast lesions. Am J Clin Pathol 2000;114:629-59.

10. Becker RL, Specht CS, Jones R, et al. Use of remote (video) microscopy as an adjunct to neurosurgical frozen section consultation. Hum Pathol 1993;24:909-11.

11. Briscoe D, Adair CF, Thompson LD, et al. Telecytologic diagnosis of breast fine needle aspiration biopsies. Intraobserver concordance. Acta Cytol 2000;44:175-80.

12. Winokur TS, McClellan S, Siegal GP, et al. A prospective trial of telepathology for intraoperative consultation (frozen sections). Hum Pathol 2000;31:781-5.

13. Ito H, Shomori K, Adachi H, Tanimaya K. Telepathology for the biopsy specimens from human allografted kidney: effectiveness and pitfalls. Clin Transplant 2000;15 (Suppl 5):5558.

14. Fisher SI, Nandekar MA, Williams BH, Abbondanzo SL. Telehematopathology in a clinical consultative practice. Hum Pathol 2001;32:1327-33.

15. Allen EA, Ollayos CW, Tellado MV, et al. Characteristics of a telecytology consultative service. Hum Pathol 2001;32:1323-6.

16. Szymas J, Wolf G, Papierz W, et al. Online internet-based robotic telepathology in the diagnosis of neuro-oncology cases: a telepathology feasibility study. Hum Pathol 2001;32: 1304-8.

17. Dunn BE, Choi H, Almagro UA, et al. Routine surgical telepathology in the Department of Veterans Affairs: experiencerelated improvements in pathologist performance in 2200 cases. Telemed J 1999;1999:5:323-37.

18. Harper R, Reeves B. Reporting of precision of estimates for diagnostic accuracy: a review. BMJ 1999;318:1322-33.

19. Bauermeister DE. The role and limitations of frozen section and needle aspiration biopsy in breast cancer diagnosis. Cancer 1980;46:947-9.

20. Remsen KA, Lucente FE, Biller HF. Reliability of frozen section diagnosis in head and neck neoplasma. Laryngoscope 1984;94:519-24.
21. Kaufman Z, Lew S, Griffel B, et al. Frozen section diagnosis in surgical pathology. A prospective analysis of 525 frozen sections. Cancer 1986;57:377-9.

22. Lessells AM, Simpson JG. A retrospective analysis of the accuracy of immediate frozen section diagnosis in surgical pathology. Br J Surg 1976;63:327-9.

23. Rogers C, Klatt EC, Chandrasoma P. Accuracy of frozen section diagnosis in a teaching hospital. Arch Pathol Lab Med 1987;111:514-7.

24. Schwan HD. Frozen section diagnosis of breast tissue. Indications and limitations. Diagn Gynecol Obstet 1981;3:347-52.

25. Granick MS, Erickson FR, Hanna DC. Accuracy of frozen section diagnosis in salivary gland lesions. Head Neck Surg 1985;7:465-7.

26. Dindzans LJ, VanNostrand AWP. The accuracy of frozen section diagnosis of parotid lesions. J Otolaryngol 1984;13:382-6.

27. Fessia L, Ghiringhello B, Arisio R, et al. Accuracy of frozen section diagnosis in breast cancer detection. A review of 4436 biopsies and comparison with cytodiagnosis. Pathol Res Pract 1984;179:61-6.

28. Wheelis RF, Yarington CT. Tumors of the salivary glands. Comparison of frozen section diagnosis with final pathologic diagnosis. Arch Otolaryngol 1984;110:76-7.

29. Della Mea V, Cataldi P, Pertoldi B, Beltrami CA. Combining dynamic and static robotic telepathology: a report on 184 consecutive cases of frozen sections, histology and cytology. Anal Cell Pathol 2000;20:33-9.

30. Baak JP, van Diest PJ, Meijer GA. Experience with a dynamic inexpensive video-conferencing system for frozen section telepathology. Anal Cell Pathol 2000;21:169-75.

31. Kayser K, Kayser G, Becker HD, Herth F. Telediagnosis of transbronchial fine needle aspirations-a feasibility study. Anal Cell Pathol 2000;21:207-12.

32. Wellnitz U, Binder B, Fritz P. Reliability of telepathology for frozen section service. Anal Cell Pathol 2000;21:213-22.

33. Goncalves L, Cunha C. One year experience with telepathology for frozen sections. Elec J Pathol Histol 1995;1:954-59.

34. Cataldi P, Pertoldi B, Della Mea V, Beltrami A. Validation of realtime telemicroscopy. A preliminary report on 184 cases. Adv Clin Pathol 1998;103:199-205.

35. Adachi H, Inoue J, Nozu T, et al. Frozen-section services by telepathology: experience of 100 cases in the San-in district, Japan. Pathol Int 1996;46:436-41. 